\title{
Article \\ Untargeted Urinary Metabolomics and Children's Exposure to Secondhand Smoke: The Influence of Individual Differences
}

\author{
Huiwei Zhu ${ }^{1,2}$, Abu S. Abdullah ${ }^{3,4,5, *}$, Jingyi He ${ }^{1,2}$, Jianxiong Xi ${ }^{1,2}$, Yimeng Mao ${ }^{1,2}$, Yitian Feng 1,2, \\ Qianyi Xiao ${ }^{1,2, *}$ and Pinpin Zheng ${ }^{1,2}$
}

1 Department of Preventive Medicine and Health Education, School of Public Health, Fudan University, Shanghai 200032, China; 18211020128@fudan.edu.cn (H.Z.); 16211020052@fudan.edu.cn (J.H.);

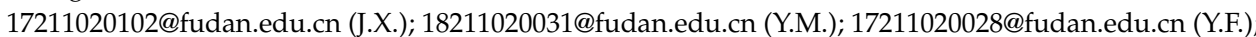
zpinpin@shmu.edu.cn (P.Z.)

2 Key Lab of Public Health Safety of the Ministry of Education and Key Lab of Health Technology Assessment of the Ministry of Health, School of Public Health, Fudan University, Shanghai 200032, China

3 Global Health Research Center, Duke Kunshan University, Kunshan 215347, China

4 Duke Global Health Institute, Duke University, Durham, NC 27710, USA

5 Department of General Internal Medicine, School of Medicine, Boston University Medical Center, Boston, MA 02118, USA

* Correspondence: xiaoqianyi@fudan.edu.cn (Q.X.); abu.abdullah@duke.edu (A.S.A.)

\section{check for} updates

Citation: Zhu, H.; Abdullah, A.S.; He, J.; Xi, J.; Mao, Y.; Feng, Y.; Xiao, Q.; Zheng, P. Untargeted Urinary Metabolomics and Children's Exposure to Secondhand Smoke: The Influence of Individual Differences. Int. J. Environ. Res. Public Health 2021, 18, 710. https://doi.org/10.3390/ ijerph18020710

Received: 5 December 2020 Accepted: 9 January 2021 Published: 15 January 2021

Publisher's Note: MDPI stays neutral with regard to jurisdictional clai$\mathrm{ms}$ in published maps and institutional affiliations.

Copyright: (C) 2021 by the authors. Licensee MDPI, Basel, Switzerland. This article is an open access article distributed under the terms and conditions of the Creative Commons Attribution (CC BY) license (https:// creativecommons.org/licenses/by/ $4.0 /)$.

\begin{abstract}
Children's exposure to secondhand smoke (SHS) is a severe public health problem. There is still a lack of evidence regarding panoramic changes in children's urinary metabolites induced by their involuntary exposure to SHS, and few studies have considered individual differences. This study aims to clarify the SHS-induced changes in urinary metabolites in preschool children by using cross-sectional and longitudinal metabolomics analyses. Urinary metabolites were quantified by using untargeted ultra high-performance liquid chromatography-mass spectrometry (UPLC (c) MS/MS). Urine cotinine-measured SHS exposure was examined to determine the exposure level. A cross-sectional study including 17 children in a low-exposure group, 17 in a medium-exposure group, and 17 in a high-exposure group was first conducted. Then, a before-after study in the cohort of children was carried out before and two months after smoking-cessation intervention for family smokers. A total of 43 metabolites were discovered to be related to SHS exposure in children in the cross-sectional analysis (false discovery rate (FDR) corrected $p<0.05$, variable importance in the projection $(\mathrm{VIP})>1.0$ ). Only three metabolites were confirmed to be positively associated with children's exposure to SHS (FDR corrected $p<0.05$ ) in a follow-up longitudinal analysis, including kynurenine, tyrosyl-tryptophan, and 1-(3-pyridinyl)-1,4-butanediol, the latter of which belongs to carbonyl compounds, peptides, and pyridines. Kyoto Encyclopedia of Genes and Genomes (KEGG) enrichment analysis indicated that 1-(3-pyridinyl)-1,4-butanediol and kynurenine were significantly enriched in xenobiotic metabolism by cytochrome P450 $(p=0.040)$ and tryptophan metabolism $(p=0.030)$, respectively. These findings provide new insights into the pathophysiological mechanism of SHS and indicate the influence of individual differences in SHS-induced changes in urinary metabolites in children.
\end{abstract}

Keywords: secondhand smoke; metabolomics; untargeted; children; cotinine

\section{Introduction}

Children's exposure to secondhand smoke (SHS) is a major public health problem, with a prevalence of $40 \%$ worldwide and $55.9 \%$ in Southeast Asia [1,2]. In China, 76.5\% of respondents from 2124 families in six counties reported smoking in front of children [3]. Compared to adults, children are more likely to be affected by exposure to SHS at the same concentration since they inhale air at relatively higher ventilation rates [4]. SHS exposure results in serious health outcomes among children aged 4-11, including asthma, 
pneumonia, middle ear disease, and impaired endothelial function, and predicts longterm atherosclerotic disease progression and cardiovascular event rates [5-9], as well as thousands of avoidable hospitalizations [10]. However, few studies have focused on SHS exposure in preschool children aged $<5$ years. These provocative findings warrant systematic investigations and early biomarker exploration for SHS-induced health problems in children.

Tobacco smoke contains more than 4000 kinds of constituents, including nicotine, tar, carbonic monoxide, polycyclic aromatic hydrocarbons, and heavy metals [11]. Nicotine is the primary component of tobacco and tobacco smoke [12]. Cotinine, which is a major metabolite of nicotine, has biological stability with a half-life of 20-30 h, while nicotine has a half-life of $2 \mathrm{~h}$ [13]. Urinary cotinine concentrations in children have been proven to indicate the degree of SHS exposure [14-18]. Studies have shown that exposure to tobacco smoke and nicotine during in utero and postnatal life impairs lung development, increases the susceptibility to lower respiratory tract infections, increases the prevalence of wheezing, and exacerbates respiratory symptoms in children with chronic lung diseases [19,20]. However, although urinary cotinine concentrations can produce a global profile of SHS exposure, they cannot provide specific clues regarding the functional pathway affected by SHS exposure and the pathophysiological mechanisms of SHS.

Metabolomics has emerged as a powerful tool for understanding metabolic changes in response to pathophysiological conditions or environmental exposures, providing an opportunity to identify biomarkers of exposure to tobacco smoke and markers that reflect host-related metabolic adaptations [21]. Several studies have indicated the value of metabolites in unraveling the biological mechanisms of the association of tobacco exposure with asthma [22], lung cancer [23], bladder cancer [24], and perinatal adverse outcomes [25]. Previous studies are largely focused on targeted metabolites caused by SHS [26,27]. In recent years, $\mathrm{Gu}$ et al. examined associations between cigarette smoking and metabolites using an untargeted metabolomics approach and identified 25 metabolites associated with smoking behaviors [28]. However, there is still a lack of knowledge on panoramic changes in urinary metabolites in response to children's exposure to SHS, and few studies have considered individual differences, such as heredity and the physiological status. In the present study, we used an untargeted metabolomics approach to explore the panoramic changes in urinary metabolites associated with children's exposure to SHS. In addition, we combined the cross-sectional and longitudinal metabolomics analyses to exclude individual differences. Specifically, differentially expressed urinary metabolites were first discovered at different levels of the SHS-exposure group in cross-sectional analyses. Then, a before-after study of the cohort of children was carried out before and two months after the smoking-cessation intervention for family smokers and the implicated urinary metabolites in cross-sectional analyses were confirmed in longitudinal analyses.

\section{Materials and Methods}

\subsection{Subject Recruitment and Sample Collection}

Subject recruitment was carried out in the Hetou and Luoyang village communities, Taizhou city, Zhejiang province, China, from 15 March 2018 to 31 March 2018. The cluster sampling method was used to select the Hetou and Luoyang village communities. Smokers were identified using the health records of community health service stations. Study coordinators went to each smoking family to describe the study. Smoking families were enrolled if they met the following criteria: (1) Smokers reported having children aged 2-5 years and smoking at home and in front of children; (2) caregivers of children were willing to provide the children's urine samples; and (3) both the smokers and the caregivers of children provided written informed consent. At the baseline, data collection was performed via an in-person questionnaire survey administered to smokers and caregivers. Data included the smoking site/venues, the smoking frequency in the past week, and the average daily cigarette amount in the past week for smokers, and the health condition of and recent respiratory symptoms among children. 
The urine samples from each child were collected in sterile fecal collection containers at home or at a community health service station and then placed in a portable refrigeration apparatus $\left(-20^{\circ} \mathrm{C}\right)$ immediately after sampling. Upon receipt, the urine samples were immediately frozen and stored at $-80^{\circ} \mathrm{C}$ until analysis.

\subsection{Ethics Statement}

This study was approved by the ethics committee of Duke Kunshan University (IRB No: 2016ABDU003). Written informed consent was obtained from each smoker and caregiver of the child.

\subsection{Follow-Up Interview}

From 15 May 2018 to 15 July 2018, we conducted smoking-cessation interventions for smokers, including health education counseling delivered by community health workers in five different sessions (two in-person and three via-telephone sessions). The counseling covered hazards about smoking and SHS exposure, tips on quitting smoking and overcoming withdrawal syndrome, how to prevent relapse, and how to ensure a smoke-free home. Follow-up interviews and assessments were conducted at 2 months after the intervention for smokers and caregivers of children. Urine samples were also collected at the follow-up.

\subsection{Cotinine Measurements, Exposure Level Stratification, and Quality Control}

Urine cotinine was analyzed using a liquid chromatography tandem mass spectrometry (LC-MS) method. Urine cotinine levels were reported in nanograms per milliliter and standardized per milligram of creatinine. Cotinine concentrations below the limit of detection (LOD, $0.5 \mathrm{ng} / \mathrm{mL}$ ) were considered unobservable and inaccurate [13,29], and were excluded. For baseline cross-sectional analysis, the children were stratified into three subgroups by cotinine concentration: Baseline low exposure (BL, 0.5-2 ng/mL); baseline medium exposure (BM, 2-10 ng/mL); and baseline high exposure (BH, 10-40 ng/mL) [18,30]. For longitudinal analysis, changes in the cotinine concentration from each child were assessed. The children at 2 months after intervention were stratified into two groups based on changes in the cotinine concentration: An intervention-no-changed (INC) group (children with a change in the cotinine concentration within $0.5 \mathrm{ng} / \mathrm{mL}$ ) and intervention-declined (ID) group (children with a declined cotinine concentration of more than $2 \mathrm{ng} / \mathrm{mL}$ ).

\subsection{Definition of SHS Exposure in This Study}

SHS exposure was determined by smokers' self-reports and by measuring the children's urinary cotinine concentrations. Positive SHS exposure was defined when both the self-reported SHS exposure in children by smokers and urine cotinine-measured SHS exposure were positive.

Children were excluded if they met the following criteria: (1) Lost to follow-up; (2) those with missing laboratory measurements of the urinary cotinine concentration; and (3) those with a cotinine concentration at the boundary value according to the cutoff value of group stratification.

\subsection{UPLC $C^{(c)}-M S / M S-B a s e d$ Urine Metabolomics}

Urine samples $(100 \mu \mathrm{L})$ were added to $300 \mu \mathrm{L}$ methanol-water $(2: 1, v / v)$ [31]. Each sample was homogenized for $1 \mathrm{~min}$, ultrasonically extracted on ice for $10 \mathrm{~min}$, stored at $-20{ }^{\circ} \mathrm{C}$ for $30 \mathrm{~min}$, and then centrifuged at 13,000 rpm for $15 \mathrm{~min}$ at $4{ }^{\circ} \mathrm{C}$. Next, $200 \mu \mathrm{L} \mathrm{su}-$ pernatant was transferred to a new vial for LC-MS/MS analysis. A mixture of all extraction aliquots was used as a quality control (QC) sample for LC-MS/MS analysis. LC-MS/MSbased urinary metabolic profiling was performed on an Ethylene Bridged Hybrid C18 column $(100 \mathrm{~mm}$ id $\times 2.1 \mathrm{~mm}, 1.7 \mu \mathrm{m}$ internal diameter, Waters Corp., Milford, MA, USA) coupled with a Triple TOF TM 5600 mass spectrometer system (AB SICEX, Framingham, MA, USA). The EBH C18 column was maintained at $45^{\circ} \mathrm{C}$ for chromatographic separation. The prepared sample was injected and maintained at $4^{\circ} \mathrm{C}$ for analysis. Samples were eluted 
using solvent A (aqueous formic acid $(0.1 \%(v / v)$ formic acid) and solvent B (acetonitrile $(0.1 \%(v / v)$ formic acid) at a flow rate of $0.40 \mathrm{~mL} / \mathrm{min}$. The separation was achieved with the following elution gradient: $1 \%$ B over $0-1 \mathrm{~min} ; 1 \% \mathrm{~B}-20 \% \mathrm{~B}$ over $1-5.5 \mathrm{~min} ; 20 \% \mathrm{~B}-30 \% \mathrm{~B}$ over 5.5-6 min; 30\% B-35\% B over 6-8.5 min; 35\% B-70\% B over 8.5-10.5 min; 70\% B-100\% B over 10.5-11 min; and the composition was held at $100 \%$ B for $2 \mathrm{~min}$, and then 13-13.1 $\mathrm{min}$, $100 \% \mathrm{~B}$ to $1 \% \mathrm{~B}$, and $13.1-15 \mathrm{~min}$ holding at $1 \% \mathrm{~B}$. The MS signal acquisition was performed in positive and negative ion scanning modes. To obtain information regarding the system repeatability, QC samples were injected at regular intervals (every six analytical samples) throughout the analytical run [32].

\subsection{Bioinformatic and Statistical Analyses}

UPLC $^{(\mathrm{c})}$-MS/MS raw data were processed using Progenesis QI software (Waters Corp, Milford, MA, USA), and a data matrix containing the retention time, mass-to-charge ratio, and peak intensity was obtained. The data matrix was preprocessed as follows: By (1) retaining variables with $>50 \%$ of nonzero values in all samples [33]; (2) filling the missing values by half of the minimum value in the original matrix to decrease the false positive results [34]; (3) normalizing the total peaks and deleting variables with Relative Standard Deviation $\geq 30 \%$ in the QC samples; and (4) performing a log10 conversion, resulting in a data matrix that was used for subsequent analysis. The mass spectra of these metabolic features were identified by using the accurate mass, MS/MS fragment spectra, and isotope ratio difference by searching in reliable biochemical databases such as the Human Metabolome Database (HMDB) (http://www.hmdb.ca/) and Metlin database (https://metlin.scripps.edu/). Concretely, the mass tolerance between the measured $\mathrm{m} / \mathrm{z}$ values and the exact mass of the components of interest was $\pm 10 \mathrm{ppm}$. For metabolites having MS/MS confirmation, only the ones with MS/MS fragment scores above 30 were considered as having been confidently identified; otherwise, metabolites only had tentative assignments. The positive and negative data were combined and imported into the SIMCA$\mathrm{P}+14.0$ software package (Umetrics, Umea, Sweden). Data analysis was performed on the Majorbio I-Sanger Cloud platform (www.i-sanger.com).

Orthogonal partial least-squares discriminant analysis (OPLS-DA) was performed using the ropls package in $\mathrm{R}$ to visually discriminate between $\mathrm{BL}$ and $\mathrm{BH}$ groups, as well as between BL and BM groups at the baseline. This approach aims to maximize the covariance between the outcome and matrix of metabolites by projecting both to linear subspaces of the original variables $[35,36]$. Validation for OPLS-DA models was conducted in a seven-fold cross-validation process, and model overfitting was examined in a 200 -fold permutation test. $\mathrm{R}^{2} \mathrm{Y}$ and $\mathrm{Q}^{2}$ were used to evaluate the goodness-of-fit and predictive ability of each model. Through an analysis of OPLS-DA loadings, taking the BL group as a reference, an independent-sample hypothesis test was used for the BH and BL groups, as well as for the $\mathrm{BM}$ and BL groups, in the cross-sectional study. The differential metabolites were identified with a variable importance in the projection (VIP) of greater than 1.0 and false discovery rate (FDR) corrected $p$ values of less than 0.05 (Student's t-test using the stats package in $R$ ). A cluster heatmap of the metabolites identified in this process was constructed using the Pheatmap package in R. The paired-sample hypothesis test (paired t-test using the stats package in R) was used to verify the differential urinary metabolites through a comparison of before intervention (pre-intervention) and after intervention (post-intervention) pairedsamples from each child in the ID and INC group in the longitudinal study. Significant differences were considered when results were below an FDR threshold of 0.05. The Kyoto Encyclopedia of Genes and Genomes (KEGG) pathway database and HMDB database were also used to uncover the predicted molecular pathways and biological functions of the metabolites. KEGG enrichment analysis of common target metabolites was performed using Fisher's exact test to obtain the significantly enriched pathways [37]. All of these analyses were performed using R version 3.6.1 (R Foundation for Statistical Computing, Vienna, Austria). and the Majorbio I-Sanger Cloud platform (www.i-sanger.com). 
Analyses of demographic data were carried out using SPSS software. (version 23.0, IBM, Chicago, USA). Continuous variables were described with the mean and standard deviation (SD), and were compared with regards to the three baseline SHS-exposure level groups by using analysis of variance (ANOVA) and the pre-intervention and postintervention groups by using a paired Student's $t$-test. Categorical variables were described with numbers and percentages, and were analyzed by Fisher's exact test in terms of the three baseline SHS-exposure level groups and McNemar's test for the pre-intervention and post-intervention groups. All tests for significance were two-sided, and $p$-values $<0.05$ were considered significant.

\section{Results}

\subsection{Study Subjects at the Baseline}

Figure 1 details the recruitment process. Among the 113 enrolled children in a smoking family at the baseline, 33 were lost to follow-up, 21 had cotinine concentrations below the LOD, and 8 had cotinine concentrations at the boundary value according to the cutoff value of group stratification. Therefore, a total of 51 preschool children aged 2-5 years who were in a smoking family were included for analysis.

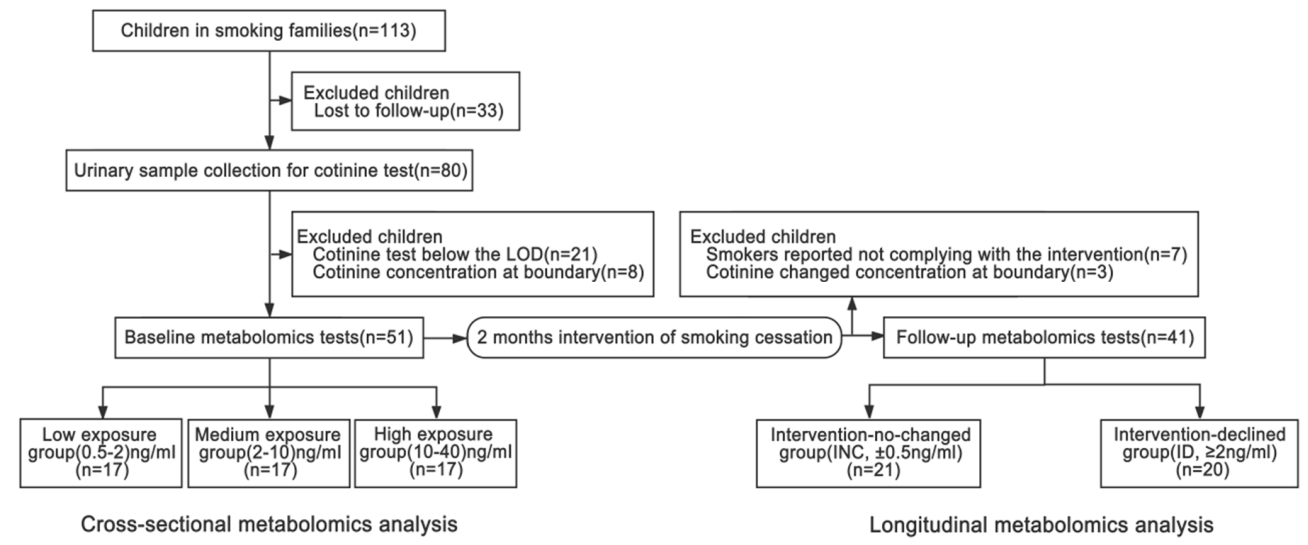

Figure 1. Flowchart of included children at the baseline and follow-up. LOD, limit of detection.

Table 1 lists the subjects' characteristics at the baseline. For family smokers, the average number of smoking days in the past week was 6.88 (SD 0.62), and the average daily cigarette amount in the past week was 19.70 (SD 10.20). There was no significant difference in the smoking frequency or smoking amount among with different SHS exposure groups defined by cotinine concentration. Among the 51 children, $88 \%$ were healthy, $18 \%$ had throat irritation or pain, $18 \%$ exhibited wheezing, and $24 \%$ had experienced nasal obstruction in the past six months, as reported by their caregivers. There was no significant difference in these symptoms among the different SHS exposure groups.

\subsection{Cross-Sectional Metabolomics Analysis at the Baseline}

The OPLS-DA score plot revealed a clear separation between high-exposure (BH) and low-exposure (BL) groups, as well as between medium-exposure (BM) and BL groups (Figure 2). Seven-fold cross-validations $\mathrm{R}^{2} \mathrm{Y}$ and $\mathrm{Q}^{2}$ indicated a good fitness, and the negative $\mathrm{Q}^{2}$ from the 200-time permutation tests suggested no overfitting in OPLS-DA models (Supplementary Table S1). At the baseline, the differentially expressed metabolites between $\mathrm{BH}$ and BL groups, as well as between BM and BL groups, at the baseline were analyzed by using the univariate t-test (predicted by the $p$ value and false discovery rate (FDR) corrected $p$ ) and multivariate OPLS-DA analysis (predicted by the variable importance in the projection (VIP)) (Table 2). Taking the BL group as a reference, 75 differential metabolites (67 upregulated/8 downregulated) in the BH group (Supplementary Table S2) and 100 differential metabolites (92 upregulated/8 downregulated) in the BM group (Supplementary Table S3) were observed in the combination mode of positive and negative ions (FDR 
corrected $p<0.05$, VIP $>1.0$ ). A total of 43 metabolites were consistently implicated in these two comparisons (BH vs. BL and BM vs. BL, Table 2), and were preliminarily identified as the metabolic biomarkers responsible for SHS exposure in baseline analyses. Additionally, a heatmap was generated to visually compare the average normalized amount of these 43 differentially expressed metabolites among the three groups (Figure 3).

Table 1. Smoking behavior of smokers and the health status of children at the baseline and follow-up.

\begin{tabular}{|c|c|c|c|c|c|c|}
\hline \multirow{2}{*}{$\begin{array}{c}\text { Baseline } \\
\text { Characteristic }\end{array}$} & \multirow[b]{2}{*}{ Total } & \multicolumn{3}{|c|}{ Cotinine Concentration } & \multirow{2}{*}{\multicolumn{2}{|c|}{$p$ Value $^{\mathrm{c}}$}} \\
\hline & & $\begin{array}{c}\text { BL }(n=17) \\
(>0.5,<2 \mathrm{ng} / \mathrm{mL})\end{array}$ & $\begin{array}{c}\mathrm{BM}(n=17) \\
(2 \sim 10 \mathrm{ng} / \mathrm{mL})\end{array}$ & $\begin{array}{r}\text { BH }(n=17) \\
(>10 \mathrm{ng} / \mathrm{mL})\end{array}$ & & \\
\hline \multicolumn{7}{|c|}{ Smokers } \\
\hline $\begin{array}{l}\text { Smoking frequency }{ }^{\mathrm{a}}, \\
\text { mean } \pm \text { SD, time }\end{array}$ & $6.88(0.62)$ & $6.65(1.03)$ & $7.00(0.00)$ & $7.00(0.00)$ & \multicolumn{2}{|c|}{0.171} \\
\hline $\begin{array}{l}\text { Cigarette amount }{ }^{b} \text {, } \\
\text { mean } \pm S D \text {, number }\end{array}$ & $19.70(10.20)$ & $16.00(8.64)$ & $20.75(10.36)$ & $22.41(10.41)$ & \multicolumn{2}{|c|}{0.172} \\
\hline \multicolumn{7}{|c|}{ Children } \\
\hline good, $n(\%)$ & $45(88)$ & $16(94)$ & $15(88)$ & $14(82)$ & \multirow{2}{*}{\multicolumn{2}{|c|}{0.567}} \\
\hline bad, $n(\%)$ & $6(12)$ & $1(6)$ & $2(12)$ & $3(18)$ & & \\
\hline \multicolumn{7}{|c|}{ Throat irritation or pain } \\
\hline yes, $n(\%)$ & $9(18)$ & $4(25)$ & $4(24)$ & $1(6)$ & \multirow{2}{*}{\multicolumn{2}{|c|}{0.276}} \\
\hline no, $n(\%)$ & $41(82)$ & $12(75)$ & $13(76)$ & $16(94)$ & & \\
\hline \multicolumn{7}{|c|}{ Wheeze } \\
\hline yes, $n(\%)$ & $9(18)$ & $1(6)$ & $3(18)$ & $5(29)$ & \multirow{2}{*}{\multicolumn{2}{|c|}{0.223}} \\
\hline no, $n(\%)$ & $41(82)$ & $15(94)$ & $14(82)$ & $12(71)$ & & \\
\hline \multicolumn{7}{|c|}{ Nasal obstruction } \\
\hline yes, $n(\%)$ & $12(24)$ & $2(12)$ & $5(29)$ & $5(29)$ & \multirow{2}{*}{\multicolumn{2}{|c|}{0.375}} \\
\hline no, $n(\%)$ & $39(76)$ & $15(88)$ & $12(71)$ & $12(71)$ & & \\
\hline \multirow{2}{*}{$\begin{array}{l}\text { Follow-up } \\
\text { characteristic }\end{array}$} & \multicolumn{3}{|c|}{$\begin{array}{l}\text { ID }(n=20) \text { (changed cotinine concentration: } \\
\qquad \geq 2 \mathrm{ng} / \mathrm{mL})\end{array}$} & \multicolumn{3}{|c|}{$\begin{array}{l}\text { INC }(n=21)(\text { changed cotinine } \\
\text { concentration: } \pm 0.5 \mathrm{ng} / \mathrm{mL})\end{array}$} \\
\hline & $\begin{array}{c}\text { Pre- } \\
\text { intervention }\end{array}$ & $\begin{array}{c}\text { Post- } \\
\text { intervention }\end{array}$ & $p$ value & $\begin{array}{c}\text { Pre- } \\
\text { intervention }\end{array}$ & $\begin{array}{c}\text { Post- } \\
\text { intervention }\end{array}$ & $p$ value \\
\hline \multicolumn{7}{|c|}{ Smokers } \\
\hline $\begin{array}{l}\text { Smoking frequency, } \\
\text { mean } \pm S D \text {, time }\end{array}$ & $7.00(0.00)$ & $3.71(3.14)$ & $0.004^{\mathrm{d}}$ & $6.88(0.48)$ & $6.59(0.84)$ & $0.082^{d}$ \\
\hline $\begin{array}{l}\text { Smoking amount, } \\
\text { mean } \pm \text { SD, number }\end{array}$ & $21.38(11.17)$ & $12.56(6.63)$ & $0.020^{\mathrm{d}}$ & $17.31(9.02)$ & $17.24(8.11)$ & $0.276^{\mathrm{d}}$ \\
\hline \multicolumn{7}{|c|}{$\begin{array}{l}\text { Children } \\
\text { Health condition }\end{array}$} \\
\hline good, $n(\%)$ & $19(95)$ & $20(100)$ & 1 non e & $20(95)$ & $19(90)$ & 1000 e \\
\hline bad, $n(\%)$ & $1(5)$ & $0(0)$ & $1.000^{e}$ & $1(5)$ & $2(10)$ & $1.000^{2}$ \\
\hline \multicolumn{7}{|c|}{ Throat irritation or pain } \\
\hline yes, $n(\%)$ & $2(10)$ & $1(6)$ & & $4(19)$ & $0(0)$ & $0.125 \mathrm{e}$ \\
\hline no, $n(\%)$ & $18(90)$ & $19(94)$ & $1.000^{\mathrm{e}}$ & $17(81)$ & $21(100)$ & $0.125^{\mathrm{e}}$ \\
\hline \multicolumn{7}{|c|}{ Wheeze } \\
\hline yes, $n(\%)$ & $4(20)$ & $0(0)$ & $0125 \mathrm{e}$ & $0(0)$ & $0(0)$ & 1 \\
\hline no, $n(\%)$ & $16(80)$ & $20(100)$ & $0.12 b^{e}$ & $21(100)$ & $21(100)$ & 1 \\
\hline \multicolumn{7}{|c|}{ Nasal obstruction } \\
\hline yes, $n(\%)$ & $4(20)$ & $0(0)$ & $0.125 \mathrm{e}$ & $3(14)$ & $1(6)$ & $0.500 \mathrm{e}$ \\
\hline no, $n(\%)$ & $16(80)$ & $20(100)$ & $0.125^{\mathrm{e}}$ & $18(86)$ & $20(94)$ & $0.500^{\mathrm{e}}$ \\
\hline
\end{tabular}

SD, standard deviation; BL, low-level secondhand smoke (SHS) exposure at the baseline; BM, medium-level SHS exposure at the baseline; BH: high-level SHS exposure at the baseline; ID group, intervention-declined group; INC, intervention-no-changed group; SD, standard deviation; ${ }^{a}$ days of smoking in the past week; ${ }^{b}$ the average daily cigarette amount in the past week; ${ }^{c}$ analysis of variance (ANOVA) for continuous variables and Fisher's exact test for categorical variables; ${ }^{\mathrm{d}} p$ value of the paired Student's $t$-test; and ${ }^{\mathrm{e}} p$ value of McNemar's test. 


\section{Positive ion}

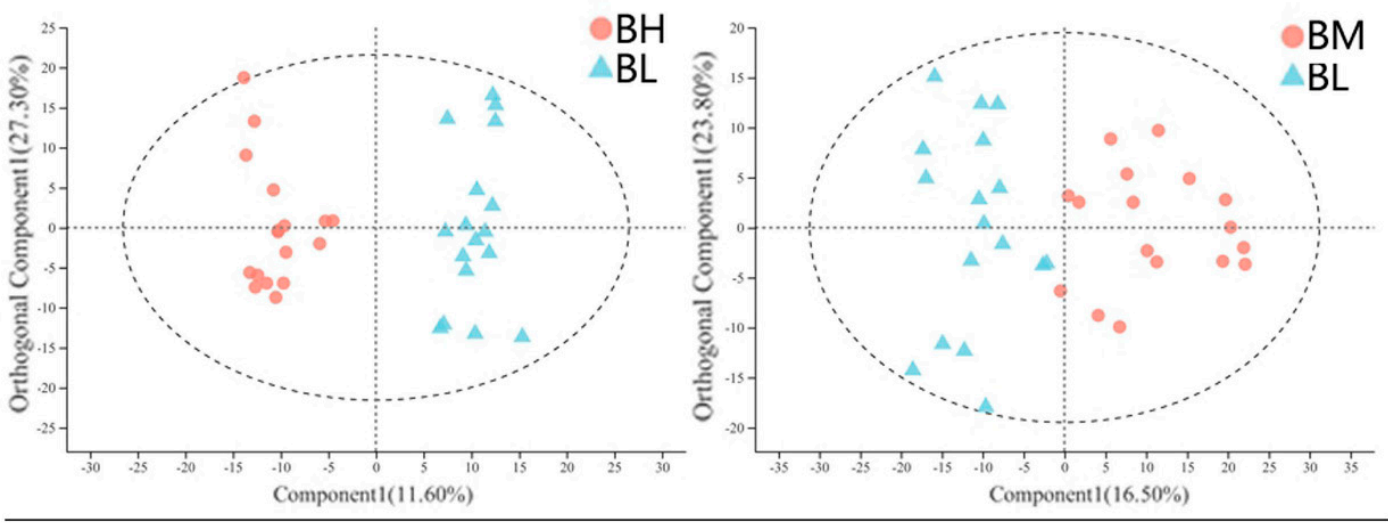

\section{Negtive ion}
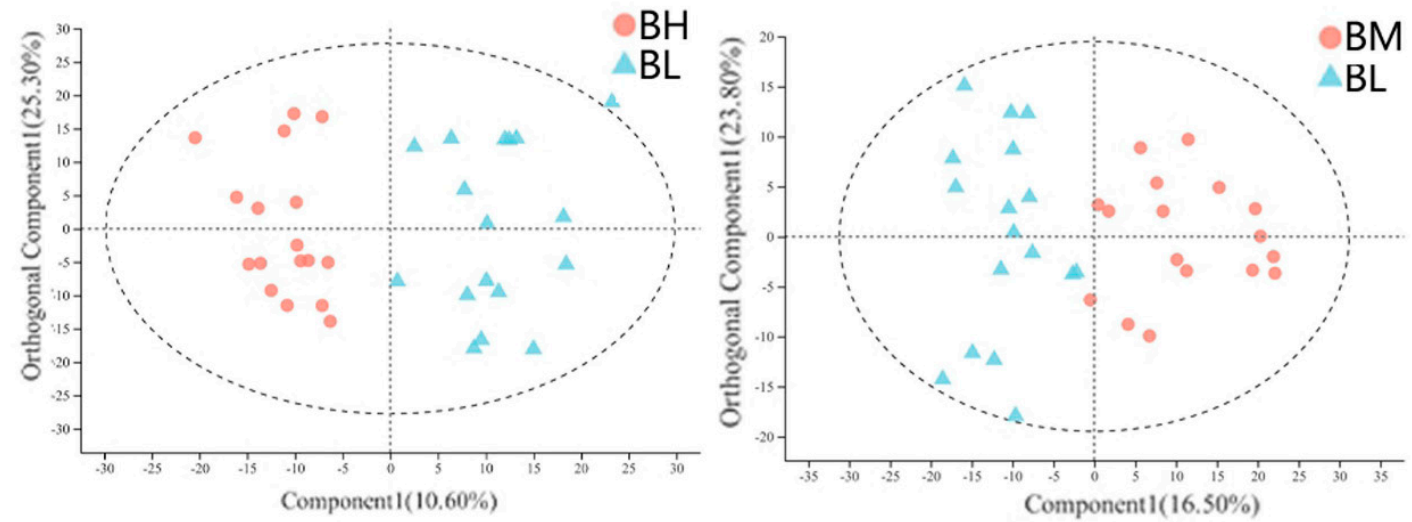

Figure 2. Orthogonal partial least-squares discriminant analysis (OPLS-DA) score plots for BH and BL groups, as well as for $\mathrm{BM}$ and BL groups, in positive-ion and negative-ion modes. Abbreviations: BH, high-level SHS exposure at the baseline; $\mathrm{BM}$, medium-level SHS exposure at the baseline; and BL, low-level SHS exposure at the baseline.

Table 2. Differential urinary metabolites at different levels of SHS exposure in children at the baseline.

\begin{tabular}{|c|c|c|c|c|c|c|c|c|}
\hline \multirow{2}{*}{ Metabolites } & \multicolumn{4}{|c|}{ BH vs. BL } & \multicolumn{4}{|c|}{ BM vs. BL } \\
\hline & VIP & FC & $p$ Value & FDR $p$ & VIP & FC & $p$ Value & FDR $p$ \\
\hline Peptides & & & & & & & & \\
\hline Valyl-Phenylalanine & 1.83 & 1.32 & $<0.001$ & 0.010 & 1.92 & 1.39 & $<0.001$ & 0.011 \\
\hline Ile Asn Asp & 1.82 & 1.45 & $<0.001$ & 0.010 & 1.52 & 1.44 & 0.003 & 0.016 \\
\hline Tyrosyl-Tryptophan & 1.74 & 2.00 & 0.002 & 0.023 & 1.67 & 1.99 & 0.007 & 0.026 \\
\hline Arg Val Asp Gly & 1.74 & 1.49 & $<0.001$ & 0.011 & 1.64 & 1.49 & $<0.001$ & 0.011 \\
\hline Asn Glu Val & 1.69 & 1.62 & 0.009 & 0.042 & 1.70 & 1.68 & 0.002 & 0.015 \\
\hline Phe Leu Gly & 1.64 & 1.62 & 0.001 & 0.018 & 1.46 & 1.53 & 0.004 & 0.018 \\
\hline Hexanoylglycine & 1.61 & 1.46 & $<0.001$ & 0.013 & 1.15 & 1.43 & 0.002 & 0.014 \\
\hline Asp Ile Glu & 1.54 & 1.49 & 0.004 & 0.028 & 1.52 & 1.46 & 0.003 & 0.017 \\
\hline Isonicotinylglycine & 1.37 & 1.11 & 0.003 & 0.028 & 1.28 & 1.10 & 0.012 & 0.036 \\
\hline Gamma-Glu-Leu & 1.35 & 1.17 & 0.004 & 0.029 & 1.31 & 1.19 & 0.003 & 0.017 \\
\hline (2S)-2-\{[1-(R)-Carboxyethyl]amino\}pentanoate & 1.34 & 1.39 & 0.012 & 0.050 & 1.37 & 1.42 & 0.006 & 0.023 \\
\hline Asp Gly Pro & 1.33 & 1.17 & 0.003 & 0.026 & 1.22 & 1.15 & 0.018 & 0.044 \\
\hline Gly Asp Tyr & 1.27 & 1.22 & 0.007 & 0.038 & 1.44 & 1.24 & 0.004 & 0.018 \\
\hline$N$-Acetylaspartylglutamic acid & 1.26 & 1.11 & 0.001 & 0.020 & 1.19 & 1.10 & 0.003 & 0.015 \\
\hline Hydroxyprolyl-Valine & 1.25 & 1.15 & 0.004 & 0.031 & 1.24 & 1.13 & 0.005 & 0.022 \\
\hline Asp Cys Arg & 1.20 & 1.22 & 0.007 & 0.037 & 1.33 & 1.22 & 0.003 & 0.016 \\
\hline Glu Val & 1.16 & 1.12 & 0.010 & 0.044 & 1.23 & 1.13 & 0.006 & 0.023 \\
\hline DL-o-Tyrosine & 1.16 & 1.49 & 0.012 & 0.050 & 1.41 & 1.65 & 0.002 & 0.014 \\
\hline
\end{tabular}


Table 2. Cont.

\begin{tabular}{|c|c|c|c|c|c|c|c|c|}
\hline \multirow{2}{*}{ Metabolites } & \multicolumn{4}{|c|}{ BH vs. BL } & \multicolumn{4}{|c|}{ BM vs. BL } \\
\hline & VIP & FC & $p$ Value & FDR $p$ & VIP & FC & $p$ Value & $\operatorname{FDR} p$ \\
\hline \multicolumn{9}{|l|}{ Lipids } \\
\hline LPA $(P-16: 0 e / 0: 0)$ & 1.97 & 1.58 & 0.002 & 0.024 & 1.58 & 1.55 & 0.009 & 0.030 \\
\hline 20-Hydroxy-leukotriene E4 & 1.65 & 1.33 & 0.001 & 0.015 & 1.77 & 1.38 & $<0.001$ & 0.011 \\
\hline Avocadyne 2-acetate & 1.64 & 1.61 & 0.003 & 0.026 & 1.53 & 1.55 & 0.011 & 0.032 \\
\hline 11-Hydroxyprogesterone 11-glucuronide & 1.52 & 1.30 & 0.007 & 0.037 & 1.51 & 1.30 & 0.015 & 0.040 \\
\hline 17-HYDROXYPROGESTERONE & 1.47 & 1.27 & $<0.001$ & 0.009 & 1.28 & 1.23 & 0.001 & 0.013 \\
\hline $\begin{array}{c}( \pm) \text {-Octanoylcarnitine } \\
\text { Carbohydrates }\end{array}$ & \multicolumn{4}{|c|}{ Carbohydrates } & & & 0.007 & 0.026 \\
\hline 2,8-Dihydroxyquinoline-beta-D-glucuronide & 2.01 & 1.57 & 0.008 & 0.040 & 1.39 & 1.52 & 0.007 & 0.025 \\
\hline \multicolumn{8}{|l|}{ Nucleosides } & 0.016 \\
\hline Deoxyadenosine & 1.47 & 1.47 & 0.004 & 0.029 & 1.63 & 1.56 & 0.003 & 0.016 \\
\hline N4-Acetylcytidine & 1.24 & 1.15 & 0.004 & 0.031 & 1.23 & 1.15 & 0.003 & 0.016 \\
\hline N6-Carbamoyl-L-threonyladenosine & 1.19 & 1.11 & 0.008 & 0.040 & 1.44 & 1.13 & 0.002 & 0.014 \\
\hline $\begin{array}{c}\text { cAMP } \\
\text { Indoles }\end{array}$ & 1.04 & 1.13 & 0.012 & 0.050 & 1.17 & 1.13 & 0.006 & 0.022 \\
\hline Indole-3-acetamide & 1.35 & 1.26 & 0.002 & 0.025 & 1.07 & 1.22 & 0.008 & 0.027 \\
\hline Indoleacrylic acid & 1.23 & 1.11 & 0.005 & 0.034 & 1.44 & 1.13 & 0.002 & 0.014 \\
\hline 5-Hydroxy-L-tryptophan & 1.23 & 1.12 & 0.002 & 0.023 & 1.28 & 1.12 & 0.002 & 0.014 \\
\hline \multicolumn{8}{|l|}{ Carbonyl compounds } & 0.042 \\
\hline \multicolumn{8}{|l|}{ Pyridines } & 0.014 \\
\hline \multicolumn{9}{|l|}{ Piperidines } \\
\hline \multicolumn{8}{|l|}{ Flavonoid glycosides } & 0.014 \\
\hline $\begin{array}{l}\text { Kaempferol 3-rhamnoside 7-xyloside } \\
\text { Amines }\end{array}$ & \multicolumn{5}{|c|}{ Amines } & 1.60 & 0.001 & 0.043 \\
\hline \multicolumn{9}{|l|}{ Alcohols } \\
\hline \multicolumn{9}{|l|}{ Not known } \\
\hline Dimethylmaleic acid anhydride & 1.39 & 1.24 & 0.002 & 0.023 & 1.31 & 1.24 & 0.004 & 0.018 \\
\hline$(R)-(+)-2-P y r r o l i d o n e-5-c a r b o x y l i c$ acid & 1.08 & 1.08 & 0.003 & 0.028 & 1.00 & 1.07 & 0.003 & 0.016 \\
\hline 4-formyl Indole & 1.04 & 1.16 & 0.007 & 0.038 & 1.15 & 1.13 & 0.014 & 0.038 \\
\hline
\end{tabular}

BH, high-level SHS exposure at the baseline; BM, medium-level SHS exposure at the baseline; BL, low-level SHS exposure at the baseline; VIP, variable importance in the projection scores; FC (BH/BL), fold change, as determined by average relative quantitation obtained from the $\mathrm{BH}$ group/BL group, where a value of less than 1 indicates a decrease in the metabolites of group $\mathrm{BH}$; FC(BM/BL), fold change, as determined by average relative quantitation obtained from the BM group/BL group, where a value of less than 1 indicates a decrease in the metabolites of group BM; and FDR $p$, false discovery rate corrected $p$ value.

The color of each section represents the abundance value of metabolite calculated by the relative quantitation normalization method. Each row corresponds to data for a specific metabolite, and each column represents the BL, BM, or BH group. Different colors represent the different intensity levels of metabolites.

\subsection{Study Subjects at Follow-Up}

As shown in Figure 1, of the 51 children at the baseline, 10 children were excluded at 2 months after intervention due to the following: (a) Smokers reported not complying with the intervention (seven children) and (b) children's cotinine variation was at the boundary value of stratification at follow-up (three children). Therefore, 41 children were included in the longitudinal analysis, with 21 children showing an unchanged cotinine concentration within the range of $0.5 \mathrm{ng} / \mathrm{mL}$ and defined as the intervention-no-changed (INC) group, and 20 children showing a declined cotinine concentration of more than $2 \mathrm{ng} / \mathrm{mL}$ and defined as the intervention-declined (ID) group. 
The follow-up data are presented in Table 1. For family smokers in the ID group, the smoking frequency decreased from 7.00 (SD 0.00) to 3.71 (SD 3.14) (paired Student's $t$-test, $p=0.004$ ), and the smoking amount decreased from 21.38 (SD 11.17) to 12.56 (SD 6.63) (paired Student's $t$-test, $p=0.020$ ). As expected, there was no significant difference in the smoking frequency and smoking amount before and after the intervention in the INC group $(p>0.050)$. For children's overall health status and respiratory symptoms reported by their caregivers, there was no significant difference before and after the intervention in both the ID and INC groups.

\section{Heatmap of Expresion Profile}
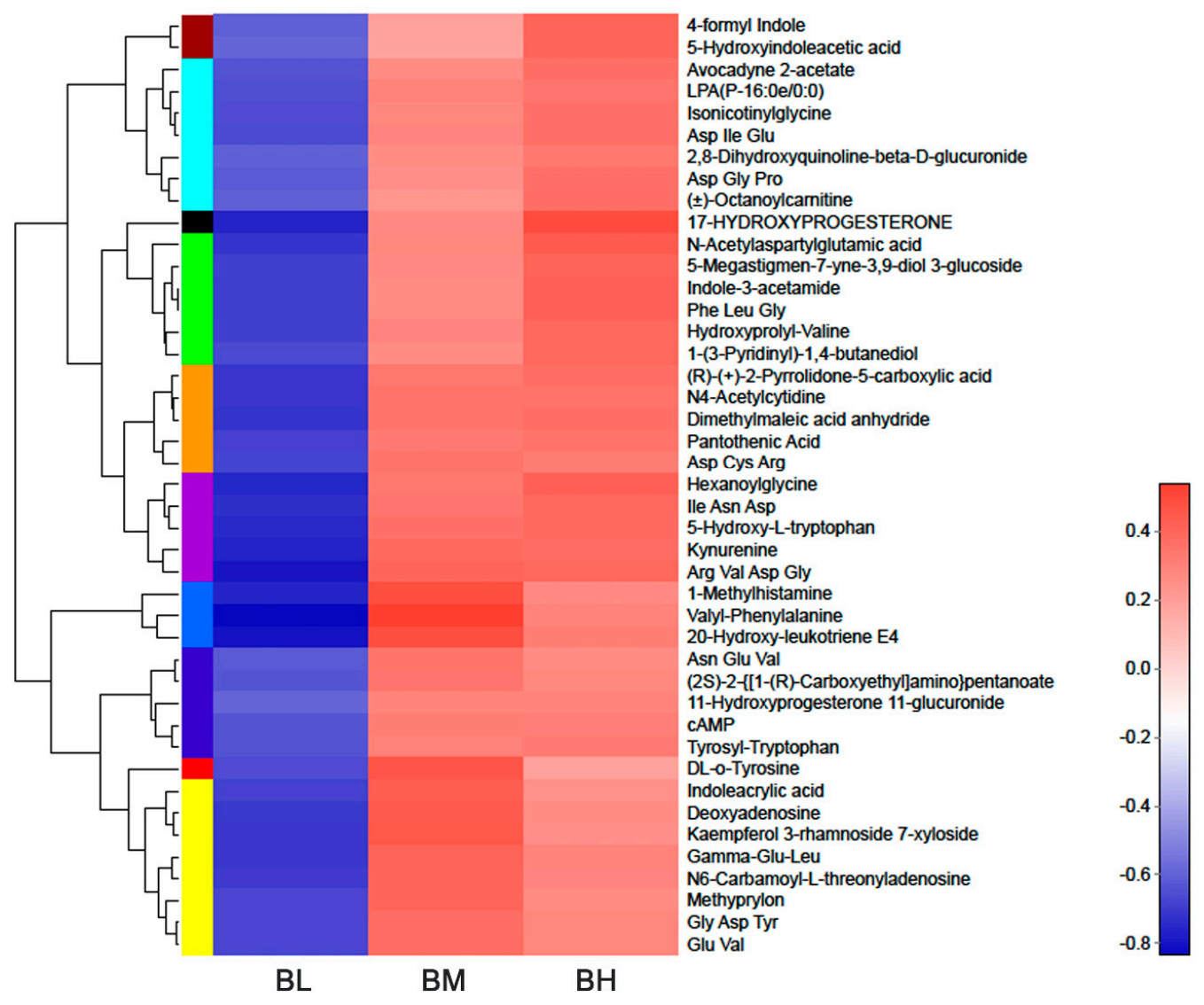

Figure 3. Heatmap of the levels of differentially expressed metabolites in each group at the baseline.

\subsection{Longitudinal Metabolomics Analysis at Follow-Up}

For the 43 differential metabolites implicated in the cross-sectional analysis, a paired t-test was performed to verify the differential urinary metabolites through a comparison of pre-intervention and post-intervention paired-samples from each child in the ID group and INC group, respectively. The metabolites showing significant differences in the ID group (FDR corrected $p<0.05$ ) while no change in the INC group (FDR corrected $p>0.05$ ) after 2 months of intervention were finally identified as the exact urinary metabolic biomarkers of SHS exposure. Details of the paired comparison for the 43 metabolites are shown in Supplementary Table S4. Table 3 displays the three metabolites confirmed to be related to SHS exposure in the longitudinal analysis, including tyrosyl-tryptophan, 1-(3pyridinyl)-1,4-butanediol, and kynurenine. The corresponding decreases in the ID group were 0.42 -fold for the tyrosyl-tryptophan level (FDR corrected $p=0.011$ in the ID group and FDR corrected $p=1.000$ in the INC group), 0.69-fold for the 1-(3-pyridinyl)-1,4-butanediol level (FDR corrected $p=0.009$ in the ID group and FDR corrected $p=1.000$ in the INC group), and 0.78-fold for the kynurenine level (FDR corrected $p=0.036$ in the ID group 
and FDR corrected $p=1.000$ in the INC group). Additionally, Figure 4 visually depicts the specific association trend of these three metabolites with the cotinine concentration in each sample of 20 children from the ID group. With the decrease of the cotinine concentration after intervention for smokers, the tyrosyl-tryptophan level declined in $65 \%(13 / 20)$ of samples, 1-(3-pyridinyl)-1,4-butanediol level declined in 90\% (18/20) of samples, and kynurenine level declined in $90 \%(18 / 20)$ of samples.

Table 3. Paired $t$-test for final differential urinary metabolites conducted through a comparison of pre-intervention and post-intervention paired-samples from each child in the ID group and INC group, respectively.

\begin{tabular}{ccccccc}
\hline \multirow{2}{*}{ Metabolites } & \multicolumn{3}{c}{ ID Group } & \multicolumn{3}{c}{ INC Group } \\
\cline { 2 - 7 } & FC (Post/Pre) & $\boldsymbol{p}$ Value & FDR $\boldsymbol{p}$ & FC (Post/Pre) & $p$ Value & FDR $\boldsymbol{p}$ \\
\hline Tyrosyl-Tryptophan & 0.42 & $<0.001$ & 0.011 & 1.26 & 0.174 & 1.000 \\
1-(3-Pyridinyl)-1,4-butanediol & 0.69 & $<0.001$ & 0.009 & 1.02 & 0.853 & 1.000 \\
Kynurenine & 0.78 & $<0.001$ & 0.036 & 1.02 & 0.694 & 1.000 \\
\hline
\end{tabular}

ID group, intervention-declined group; INC, intervention-no-changed group; FC, fold change; and FDR $p$, false discovery rate corrected $p$ value.

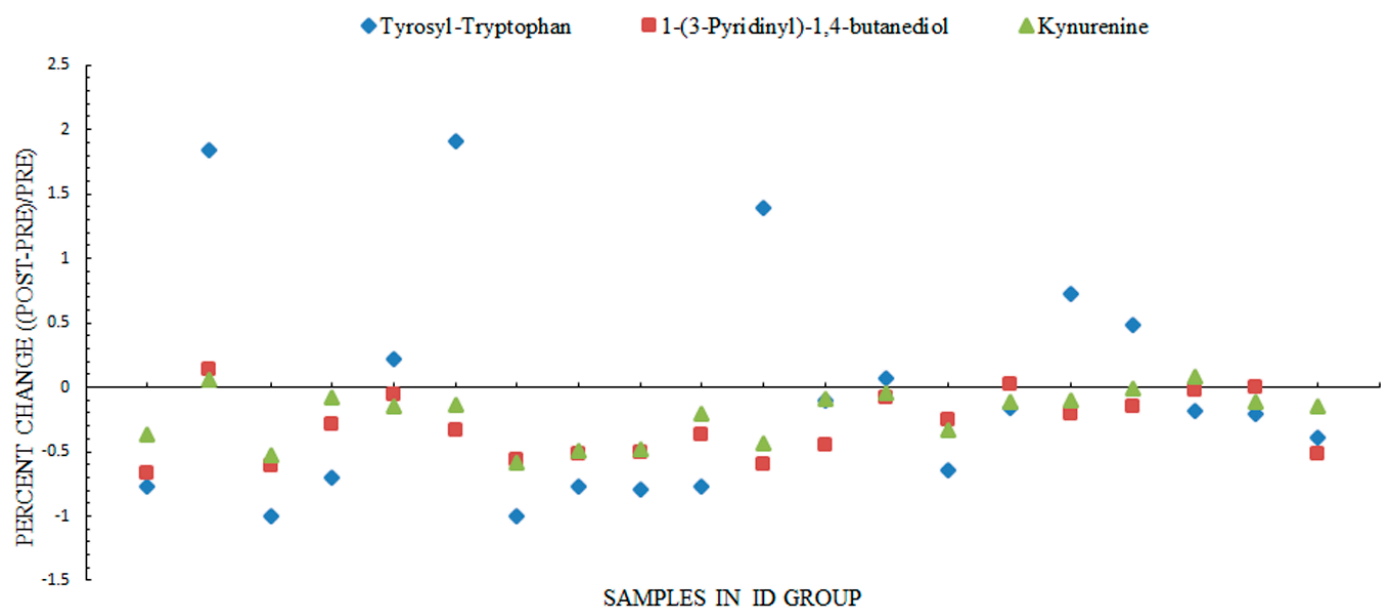

Figure 4. Percent change of the three differential metabolites at post-intervention compared to pre-intervention for each child from the ID group. Blue, tyrosyl-tryptophan; red, 1-(3-pyridinyl)-1,4-butanediol; green, kynurenine; and ID, concentration of nicotine in children's urine was decreased after the smoking-cessation intervention for smokers, which is called the intervention-declined group (ID) for short.

\subsection{Metabolic Pathway Analysis}

As shown in Table 4, KEGG pathway enrichment analysis suggested that 1-(3-pyridin yl)-1,4-butanediol was significantly enriched in xenobiotic metabolism by cytochrome P450 $(p=0.040)$, and kynurenine was significantly enriched in tryptophan metabolism $(p=0.030)$. Figure 5 depicts a simplified schematic of the involved metabolism pathway of these two metabolites following the KEGG pathway database.

Table 4. The metabolic pathways for identified urinary metabolic biomarkers.

\begin{tabular}{|c|c|c|c|c|}
\hline Metabolite & Molecular Formula & Library ID ${ }^{a}$ & Related Pathway & $p$ Value $^{\mathrm{b}}$ \\
\hline Tyrosyl-Tryptophan & $\mathrm{C} 20 \mathrm{H} 21 \mathrm{~N} 3 \mathrm{O} 4$ & HMDB0029116 & Not Known & - \\
\hline 1-(3-Pyridinyl)-1,4-butanediol & $\mathrm{C} 9 \mathrm{H} 13 \mathrm{NO} 2$ & HMDB0062266 & $\begin{array}{l}\text { Metabolism of xenobiotics by } \\
\text { cytochrome P450 }\end{array}$ & 0.040 \\
\hline Kynurenine & $\mathrm{C} 10 \mathrm{H} 12 \mathrm{~N} 2 \mathrm{O} 3$ & HMDB0000684 & Tryptophan metabolism & 0.030 \\
\hline
\end{tabular}

${ }^{a}$ Identity from the human metabolite database (HMDB) database. ${ }^{\mathrm{b}} p$ value calculated from the Kyoto Encyclopedia of Genes and Genomes (KEGG) pathway enrichment analysis. Criteria: $p$ value $<0.05$ was defined as significantly enriched. 


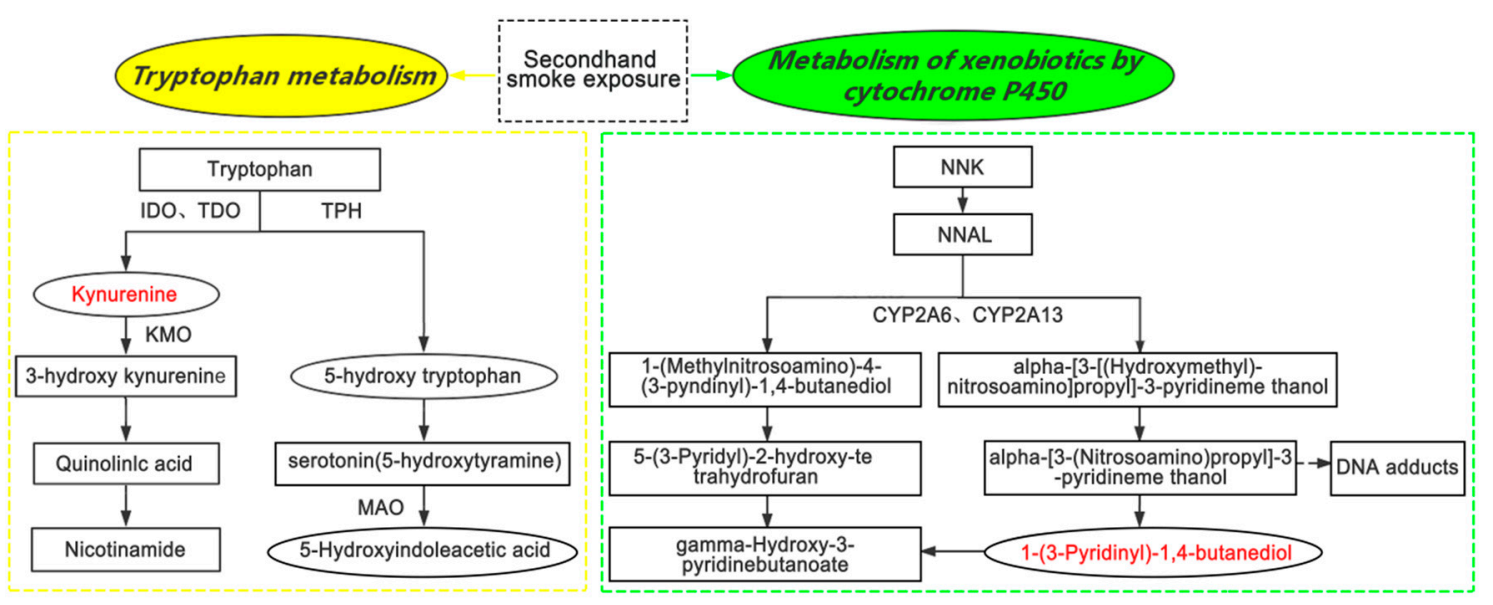

Figure 5. Simplified schematic of the relationship between exposure to SHS and metabolites following the KEGG pathway database.

Red represents the metabolites reported in the present study, which showed a positive relation with SHS exposure. The dotted arrows indicate indirect reactions. Abbreviations: IDO, indoleamine 2,3-dioxygenase; MAO, monoamine oxidase; TDO, tryptophan 2,3dioxygenase; TPH, tryptophan hydroxylase; KMO, kynurenine 3-monooxygenase; NNK, 4-(methylnitrosamino)-1-(3-pyridyl)-1-butanone; NNAL, 4-(N-nitrosomethylamino)-1-(3pyridyl)-1-butanol; and CYP, Cytochrome P450.

\section{Discussion}

To the best of our knowledge, this is the first study to combine cross-sectional and longitudinal metabolomics to clarify the SHS-induced changes in urinary metabolites in children. Three metabolic biomarkers, including tyrosyl-tryptophan, 1-(3-pyridinyl)-1,4butanediol, and kynurenine, were identified as being positively associated with cotinine exposure in children. Among these three urinary metabolites, it is the first time that tyrosyl-tryptophan and 1-(3-pyridinyl)-1,4-butanediol have been reported to be related to SHS exposure.

The results from our study suggested a positive relation between kynurenine and tyrosyl-tryptophan and SHS exposure. The kynurenine pathway is responsible for tryptophan metabolism, and 95\% of tryptophan is metabolized via the kynurenine pathway [38]. Therefore, our results suggest a pivotal relationship between tryptophan/kynurenine metabolism and children's exposure to SHS. A previous study including 20 prospective cohorts from the US, Europe, Australia, and Asia showed that the highest quintiles of serum kynurenine were associated with a $22-31 \%$ higher risk of lung cancer compared with the lowest quintiles [39]. Additionally, the downstream metabolites of kynurenine, including 3-hydroxykynurenine $(3 \mathrm{HK})$ and quinolinic acid, are potently neurotoxic and attributed to major neurodegenerative diseases, such as schizophrenia, Alzheimer's disease, Huntington's disease, bipolar disorder, and depression [40-42]. Such an adverse role of kynurenine is furthered here by implicating its positive association with children's exposure to SHS in our study, indicating the potential risk of future disease in children exposed to SHS for a long period of time. Oades et al. [43] explored whether the levels of cytokines and tryptophan metabolites were associated with features of the index pregnancy of potential etiological significance and found that increased maternal smoking during pregnancy was associated with decreasing kynurenine levels in attention-deficit hyperactivity disorder (ADHD) children, but increasing kynurenine levels in controls, which is consistent with our findings. However, there are also studies showing the opposite conclusion. Naz et al. found that in chronic obstructive pulmonary disease (COPD) patients, the level of kynurenine, which is the main product of tryptophan [44], decreased in smokers relative 
to never-smokers [38]. Mathai et al. [45] reported that in schizophrenic patients, current smokers showed lower kynurenine levels than past smokers, which further elucidates the neurobiological underpinnings of altered kynurenine levels in smokers. In these studies, there were non-significant decreases in tryptophan levels. Therefore, the lower kynurenine in schizophrenic patients may be due to the decreased dietary intake in schizophrenic patients (broadly reported in smokers) [46]. It is supposed that people with specific diseases may show different change patterns in metabolites in reaction to SHS exposure due to factors such as the disease itself [47], diet, medication, physiological status, and so on.

We also found a positive relation between tyrosyl-tryptophan and the cotinine concentration. Tryptophan has been extensively studied, but evidence of tyrosyl-tryptophan is sparse. Tryptophan is a nutritionally essential amino acid for both humans and animals. In addition to acting as a building block for protein synthesis, tryptophan and its metabolites are crucial for maintaining neurological function, immunity, and homeostasis in the body [48]. Paternal smoking during maternal pregnancy was related to increased tryptophan in control children compared to children with ADHD [43].

Our study is the first to reveal a positive correlation between urine 1-(3-pyridinyl)1,4-butanediol and the cotinine concentration. As shown in Figure 5, 1-(3-pyridinyl)-1,4butanediol participates in the 4-(N-nitrosomethylamino)-1-(3-pyridyl)-1-butanol (NNAL) pathway and serves as a downstream metabolite of NNAL. NNAL is the most potent tobacco-specific carcinogen, and it has been frequently used as a biomarker to assess human smoke exposure [49]. Both urinary cotinine and NNAL are sensitive and specific biomarkers for discriminating the source of tobacco smoke exposure. The half-life of NNAL (10-16 days) is much longer than that of cotinine (16 h), suggesting that NNAL might be a better measurement of tobacco exposure over time and that NNAL might be a better biomarker when sampling cannot be done in temporal proximity to tobacco smoke exposure [50]. Our data from untargeted metabolomics suggested that 1-(3-pyridinyl)-1,4butanediol may be a supplementary biomarker of NNAL for long-term exposure to SHS.

A previous study on metabolites relevant to children's exposure to SHS mainly focused on targeted metabolomics. A randomized clinical trial in 195 female smokers with children aged $\leq 10$ years residing in their homes aimed to promote smoke-free homes through biomarker feedback documenting a child's exposure to tobacco toxins, documenting the levels of nicotine, cotinine, and NNAL [51]. A previous study in children living in homes of hookah-only smokers and nonsmokers examined the child uptake of nicotine, the carcinogen 4-(methylnitrosamino)-1-(3-pyridyl)-1-butanone (NNK), and the toxicant acrolein by analyzing their corresponding metabolites cotinine, 4-(methylnitrosamino)-1-(3-pyridyl)-1butanol (NNAL) and NNAL-glucuronides (total NNAL), and 3-hydroxypropylmercapturic acid, and the results provide evidence for the uptake of nicotine, the tobacco-specific lung carcinogen NNK, and the ciliatoxic and cardiotoxic agent acrolein in children living in homes of hookah smokers compared with nonsmokers [52]. Although recent studies used the untargeted metabolomics method to explore smoking-relevant metabolites, they only focused on active adult smoking. Garcia-Perez et al. applied CE-MS to a metabolomics analysis of human urine from cigarette smokers and nonsmokers and detected significant changes in urinary glycine and serine, which are intermediates in the metabolism of glutathione in cigarette smokers and nonsmokers [53]. Seow et al. performed a prospective study to examine the association between untargeted urinary metabolomics and the risk of lung cancer among women in China and reported that an increased level of urinary 5-methyl-2-furoic acid was associated with a decreased risk of lung cancer [54]. Such an application of untargeted metabolomics is furthered here by implicating urinary metabolomic changes that are associated with children's exposure to SHS.

Several limitations of this study should be acknowledged. First, although we combined the cross-sectional baseline comparison with the longitudinal pre-intervention and post-intervention comparison to remove the influence of individual differences and improve the reliability of the results, this study included a preliminary analysis with a small sample size, and studies of larger populations with different groups of children across 
Asia or with different ethnicities would give a better picture of the connection between urinary metabolites and SHS exposure. Second, the urine samples were only collected once, and we were unable to assess the temporal trends of metabolites over time, making it impossible to derive the long-term effects of metabolites on children with SHS exposure. Subsequent studies should measure urine samples multiple times to obtain long-term influence trends of metabolites. Third, although our study suggests tyrosyl-tryptophan, 1-(3-pyridinyl)-1,4-butanediol, and kynurenine as urinary metabolic biomarkers for SHS exposure, which may be involved in the pathogenesis of disease of the respiratory system, further work, including long-term observations of children and animal experiments, will be needed to determine whether these urinary metabolic biomarkers are predictors of long-term illness. Fourthly, the study lacks urine samples from control children, who were not exposed to second-hand smoke, because this study was based on the smoking-cessation intervention program and because it is difficult to obtain preschool children's samples and information in nonsmoking families. However, we set low, medium, and high urine cotinine levels and treated the low-concentration group as the control group to perform multiple comparison analyses, in order to address this limitation. Fifthly, we did not test the markers of kidney function in urine, which may have some influence on the level of cotinine and metabolites in urine. Further research with elaborate markers of kidney function in urine is needed to confirm the SHS-associated metabolites identified in our study. Finally, although, in metabolomics work, it is common to use a criterion of a $50 \%$ rule to retain the metabolites with missing intensity values in no more than $50 \%$ of the samples for statistical analysis [33], the imputation of missing data with the $50 \%$ criterion may be risky and a comparison of the values with those of other imputation strategies is needed in analysis, such as random forests [55].

\section{Conclusions}

In summary, this study is the first to have combined cross-sectional and longitudinal metabolomics analysis to examine urinary biomarkers in response to children's SHS exposures. In addition to the well-established nicotine metabolite kynurenine, we newly identified tyrosyl-tryptophan and 1-(3-pyridinyl)-1,4-butanediol as potential metabolic markers and related functional pathways affected by SHS in children, contributing new insights into the pathophysiological mechanism of SHS. Importantly, our findings indicated that individual differences should be taken into account when exploring urinary metabolites related to children's exposure to SHS.

Supplementary Materials: The following are available online at https:/ / www.mdpi.com/1660-460 1/18/2/710/s1, Table S1: Model validation and permutation results for OPLS-DA models, Table S2: Metabolites with statistically significant differences between baseline high with baseline low group, Table S3: Metabolites with statistically significant differences between baseline medium with baseline low group, Table S4: Paired t-test to verify 43 urinary metabolites discovered at baseline through the comparison between pre-intervention and post-intervention paired-samples from each child at ID group and INC group respectively.

Author Contributions: Q.X., P.Z., and A.S.A. designed the study. H.Z., J.H., J.X., Y.M., and Y.F. were responsible for data collection. H.Z. and J.X. performed the statistical analyses. Q.X. and H.Z. drafted the manuscript. P.Z. and A.S.A. reviewed the manuscript. All authors have read and agreed to the published version of the manuscript.

Funding: This study was supported by the National Natural Science Foundation of China (NSFC) [Award number: 71673125] and grant from Shanghai Municipal Committee for Health and Family Planning (Award number: GWV-10.1-XK14).

Institutional Review Board Statement: The study was conducted according to the guidelines of the Declaration of Helsinki, and approved by the Ethics Committee of Duke Kunshan University (IRB No: 2016ABDU003).

Informed Consent Statement: Informed consent was obtained from each smoker and caregivers of the child involved in the study. 
Data Availability Statement: The data that support the findings of this study are available from the corresponding author upon reasonable request. Restrictions apply to public availability of these data due to confidential agreement included in the consent form.

Acknowledgments: The authors thank all the study coordinators, research nurses, and lab technicians for their efforts related to this study, and all of the participants for their cooperation.

Conflicts of Interest: The authors declare no conflict of interest.

\section{References}

1. Oberg, M.; Jaakkola, M.S.; Woodward, A.; Peruga, A.; Prüss-Ustün, A. Worldwide burden of disease from exposure to second-hand smoke: A retrospective analysis of data from 192 countries. Lancet 2011, 377, 139-146. [CrossRef]

2. Xi, B.; Liang, Y.; Liu, Y.; Yan, Y.; Zhao, M.; Ma, C.; Bovet, P. Tobacco use and second-hand smoke exposure in young adolescents aged 12-15 years: Data from 68 low-income and middle-income countries. Lancet Glob. Health 2016, 4, e795-e805. [CrossRef]

3. Wang, C.P.; Ma, S.J.; Xu, X.F.; Wang, J.F.; Mei, C.Z.; Yang, G.H. The prevalence of household second-hand smoke exposure and its correlated factors in six counties of China. Tob. Control 2009, 18, 121-126. [CrossRef] [PubMed]

4. Hwang, S.H.; Hwang, J.H.; Moon, J.S.; Lee, D.H. Environmental tobacco smoke and children's health. Korean J. Pediatrics 2012, 55, 35-41. [CrossRef] [PubMed]

5. Sims, M.; Bauld, L.; Gilmore, A. England's legislation on smoking in indoor public places and work-places: Impact on the most exposed children. Addiction 2012, 107, 2009-2016. [CrossRef]

6. Strachan, D.P.; Cook, D.G. Health effects of passive smoking. 6. Parental smoking and childhood asthma: Longitudinal and case-control studies. Thorax 1998, 53, 204-212. [CrossRef]

7. Strachan, D.P.; Jarvis, M.J.; Feyerabend, C. Passive smoking, salivary cotinine concentrations, and middle ear effusion in 7 year old children. BMJ 1989, 298, 1549-1552. [CrossRef]

8. Kallio, K.; Jokinen, E.; Raitakari, O.T.; Hämäläinen, M.; Siltala, M.; Volanen, I.; Kaitosaari, T.; Viikari, J.; Rönnemaa, T.; Simell, O. Tobacco smoke exposure is associated with attenuated endothelial function in 11-year-old healthy children. Circulation 2007, 115, 3205-3212. [CrossRef]

9. Schachinger, V.; Britten, M.B.; Zeiher, A.M. Prognostic impact of coronary vasodilator dysfunction on adverse long-term outcome of coronary heart disease. Circulation 2000, 101, 1899-1906. [CrossRef]

10. Goldstein, A.O. Is exposure to secondhand smoke child abuse? Yes. Ann. Fam. Med. 2015, 13, 103-104. [CrossRef]

11. Dai, J.B.; Wang, Z.X.; Qiao, Z.D. The hazardous effects of tobacco smoking on male fertility. Asian J. Androl. 2015, 17, 954-960.

12. Siqueira, L.M. Nicotine and Tobacco as Substances of Abuse in Children and Adolescents. Pediatrics 2017, 139, e20163436. [CrossRef] [PubMed]

13. Ino, T.; Ohtani, T.; Yoshimi, I. Urinary biomarkers for secondhand smoke. J. Clin. Lab. Anal. 2011, 25, 354-358. [CrossRef] [PubMed]

14. Kalliola, S.; Pelkonen, A.S.; Malmberg, L.P.; Sarna, S.; Hämäläinen, M.; Mononen, I.; Mäkelä, M.J. Maternal smoking affects lung function and airway inflammation in young children with multiple-trigger wheeze. J. Allergy Clin. Immunol. 2013, 131, 730-735. [CrossRef] [PubMed]

15. Rabinovitch, N.; Silveira, L.; Gelfand, E.W.; Strand, M. The response of children with asthma to ambient particulate is modified by tobacco smoke exposure. Am. J. Respir. Crit. Care Med. 2011, 184, 1350-1357. [CrossRef]

16. Park, S.; Cho, S.C.; Hong, Y.C.; Kim, J.W.; Shin, M.S.; Yoo, H.J.; Han, D.H.; Cheong, J.H.; Kim, B.N. Environmental tobacco smoke exposure and children's intelligence at 8-11 years of age. Environ. Health Perspect. 2014, 122, 1123-1128. [CrossRef]

17. Sexton, K.; Adgate, J.L.; Church, T.R.; Hecht, S.S.; Ramachandran, G.; Greaves, I.A.; Fredrikson, A.L.; Ryan, A.D.; Carmella, S.G.; Geisser, M.S. Children's exposure to environmental tobacco smoke: Using diverse exposure metrics to document ethnic/racial differences. Environ. Health Perspect. 2004, 112, 392-397. [CrossRef]

18. Hovell, M.F.; Wahlgren, D.R.; Liles, S.; Jones, J.A.; Hughes, S.C.; Matt, G.E.; Ji, M.; Lessov-Schlaggar, C.N.; Swan, G.E.; Chatfield, D.; et al. Providing coaching and cotinine results to preteens to reduce their secondhand smoke exposure: A randomized trial. Chest 2011, 140, 681-689. [CrossRef]

19. Gibbs, K.; Collaco, J.M.; Mcgrath-Morrow, S.A. Impact of Tobacco Smoke and Nicotine Exposure on Lung Development. Chest 2016, 149, 552-561. [CrossRef]

20. Maritz, G.S.; Harding, R. Life-long programming implications of exposure to tobacco smoking and nicotine before and soon after birth: Evidence for altered lung development. Int. J. Environ. Res. Public Health 2011, 8, 875-898. [CrossRef]

21. Fiehn, O. Metabolomics-The link between genotypes and phenotypes. Plant Mol. Biol. 2002, 48, 155-171. [CrossRef] [PubMed]

22. Lang, J.E.; Dozor, A.J.; Holbrook, J.T.; Mougey, E.; Krishnan, S.; Sweeten, S.; Wise, R.A.; Teague, W.G.; Wei, C.Y.; Shade, D.; et al. Biologic mechanisms of environmental tobacco smoke in children with poorly controlled asthma: Results from a multicenter clinical trial. J. Allergy Clin. Immunol. Pract. 2013, 1, 172-180. [CrossRef] [PubMed]

23. Hecht, S.S.; Stepanov, I.; Carmella, S.G. Exposure and Metabolic Activation Biomarkers of Carcinogenic Tobacco-Specific Nitrosamines. Acc. Chem. Res. 2016, 49, 106-114. [CrossRef] [PubMed] 
24. Jin, F.; Thaiparambil, J.; Donepudi, S.R.; Vantaku, V.; Piyarathna, D.W.B.; Maity, S.; Krishnapuram, R.; Putluri, V.; Gu, F.; Purwaha, P.; et al. Tobacco-Specific Carcinogens Induce Hypermethylation, DNA Adducts, and DNA Damage in Bladder Cancer. Cancer Prev. Res. 2017, 10, 588-597. [CrossRef]

25. Dessì, A.; Corona, L.; Pintus, R.; Fanos, V. Exposure to tobacco smoke and low birth weight: From epidemiology to metabolomics. Expert Rev. Proteom. 2018, 15, 647-656. [CrossRef] [PubMed]

26. Wang-Sattler, R.; Yu, Y.; Mittelstrass, K.; Lattka, E.; Altmaier, E.; Gieger, C.; Ladwig, K.H.; Dahmen, N.; Weinberger, K.M.; Hao, P.; et al. Metabolic profiling reveals distinct variations linked to nicotine consumption in humans-First results from the KORA study. PLOS ONE 2008, 3, e3863. [CrossRef]

27. Müller, D.C.; Degen, C.; Scherer, G.; Jahreis, G.; Niessner, R.; Scherer, M. Metabolomics using GC-TOF-MS followed by subsequent GC-FID and HILIC-MS/MS analysis revealed significantly altered fatty acid and phospholipid species profiles in plasma of smokers. J. Chromatogr. B Analyt. Technol. Biomed. Life Sci. 2014, 966, 117-126. [CrossRef]

28. Gu, F.; Derkach, A.; Freedman, N.D.; Landi, M.T.; Albanes, D.; Weinstein, S.J.; Mondul, A.M.; Matthews, C.E.; Guertin, K.A.; Xiao, Q.; et al. Cigarette smoking behaviour and blood metabolomics. Int. J. Epidemiol. 2016, 45, 1421-1432. [CrossRef]

29. Vardavas, C.I.; Fthenou, E.; Patelarou, E.; Bagkeris, E.; Murphy, S.; Hecht, S.S.; Connolly, G.N.; Chatzi, L.; Kogevinas, M. Exposure to different sources of second-hand smoke during pregnancy and its effect on urinary cotinine and tobacco-specific nitrosamine (NNAL) concentrations. Tob. Control 2013, 22, 194-200. [CrossRef] [PubMed]

30. Ino, T.; Shibuya, T.; Saito, K.; Ohshima, J.; Okada, R. A passive smoking screening program for children. Prev. Med. 2006, 42, 427-429. [CrossRef]

31. Raftery, D. (Ed.) Mass Spectrometry in Metabolomics. In Methods in Molecular Biology; Humana Press: Totowa, NJ, USA, 2014. [CrossRef]

32. Want, E.J.; Wilson, I.D.; Gika, H.; Theodoridis, G.; Plumb, R.S.; Shockcor, J.; Homes, E.; Nicholson, J.K. Global metabolic profiling procedures for urine using UPLC-MS. Nat. Protoc. 2010, 5, 1005-1018. [CrossRef] [PubMed]

33. Huan, T.; Li, L. Counting missing values in a metabolite-intensity data set for measuring the analytical performance of a metabolomics platform. Anal. Chem. 2015, 87, 1306-1313. [CrossRef]

34. Di Guida, R.; Engel, J.; Allwood, J.W.; Weber, R.J.M.; Jones, M.R.; Sommer, U.; Viant, M.R.; Dunn, W.B. Non-targeted UHPLC-MS metabolomic data processing methods: A comparative investigation of normalisation, missing value imputation, transformation and scaling. Metabolomics 2016, 12, 93. [CrossRef]

35. Antonelli, J.; Claggett, B.L.; Henglin, M.; Kim, A.; Ovsak, G.; Kim, N.; Deng, K.; Rao, K.; Tyagi, O.; Watrous, J.D.; et al. Statistical Workflow for Feature Selection in Human Metabolomics Data. Metabolites 2019, 9, 143. [CrossRef] [PubMed]

36. Barker, M.; Rayens, W. Partial least squares for discrimination. J. Chemom. 2003, 17, 166-173. [CrossRef]

37. Xu, F.; Li, X.; Niu, W.; Ma, G.; Sun, Q.; Bi, Y.; Guo, Z.; Ren, D.; Hu, J.; Yuan, F.; et al. Metabolomic profiling on rat brain of prenatal malnutrition: Implicated for oxidative stress and schizophrenia. Metab. Brain Dis. 2019, 34, 1607-1613. [CrossRef] [PubMed]

38. Naz, S.; Bhat, M.; Stahl, S.; Forsslund, H.; Skold, C.M.; Wheelock, A.M.; Wheelock, C.E. Dysregulation of the Tryptophan Pathway Evidences Gender Differences in COPD. Metabolites 2019, 9, 212. [CrossRef]

39. Huang, J.Y.; Larose, T.L.; Luu, H.N.; Wang, R.; Fanidi, A.; Alcala, K.; Steven, V.L.; Weinstein, S.J.; Albanes, D.; Caporaso, N.E.; et al. Circulating markers of cellular immune activation in prediagnostic blood sample and lung cancer risk in the Lung Cancer Cohort Consortium (LC3). Int. J. Cancer 2020, 146, 2394-2405. [CrossRef]

40. Lahiri, P.; Dhaware, D.; Singh, A.; Panchagnula, V.; Ghosh, D. Quantitation of Neurotoxic Metabolites of the Kynurenine Pathway by Laser Desorption Ionization Mass Spectrometry (LDI-MS). Methods Mol. Biol. 2019, 1996, 113-129.

41. Guillemin, G.J. Quinolinic acid, the inescapable neurotoxin. FEBS J. 2012, 279, 1356-1365. [CrossRef]

42. Castellano-Gonzalez, G.; Jacobs, K.R.; Don, E.; Cole, N.J.; Adams, S.; Lim, C.K.; Lovejoy, D.B.; Guillemin, G.J. Kynurenine 3Monooxygenase Activity in Human Primary Neurons and Effect on Cellular Bioenergetics Identifies New Neurotoxic Mechanisms. Neurotox. Res. 2019, 35, 530-541. [CrossRef] [PubMed]

43. Oades, R.D. An exploration of the associations of pregnancy and perinatal features with cytokines and tryptophan/kynurenine metabolism in children with attention-deficit hyperactivity disorder (ADHD). ADHD Atten. Deficit Hyperact. Disord. 2011, 3, 301-318. [CrossRef] [PubMed]

44. Meier, M.A.; Ottiger, M.; Vögeli, A.; Steuer, C.; Bernasconi, L.; Thomann, R.; Christ-Crain, M.; Henzen, C.; Hoess, C.; Zimmerli, W.; et al. Activation of the Serotonin Pathway is associated with Poor Outcome in COPD Exacerbation: Results of a Long-Term Cohort Study. Lung 2017, 195, 303-311. [CrossRef] [PubMed]

45. Mathai, A.J.; Kanwar, J.; Okusaga, O.; Fuchs, D.; Lowry, C.A.; Peng, X.; Giegling, I.; Hartmann, A.M.; Konte, B.; Friedl, M.; et al. Blood Levels of Monoamine Precursors and Smoking in Patients with Schizophrenia. Front. Public Health 2016, 4, 182. [CrossRef]

46. Theofylaktopoulou, D.; Ulvik, A.; Midttun, Ø.; Ueland, P.M.; Vollset, S.E.; Nygard, O.; Hustad, S.; Tell, G.S.; Eussen, S.J.P.M. Vitamins B2 and B6 as determinants of kynurenines and related markers of interferon- $\gamma$-mediated immune activation in the community-based Hordaland Health Study. Br. J. Nutr. 2014, 112, 1065-1072. [CrossRef]

47. Sorgdrager, F.; Vermeiren, Y.; Van Faassen, M.; van den Ley, C.; Nollen, E.A.A.; Kema, I.P.; de Deyn, P.P. Age- and disease-specific changes of the kynurenine pathway in Parkinson's and Alzheimer's disease. J. Neurochem. 2019, 151, 656-668. [CrossRef]

48. Dai, Z.; Sun, S.; Chen, H.; Liu, M.; Zhang, L.; Wu, Z.; Li, J.; Wu, G. Analysis of Tryptophan and Its Metabolites by HighPerformance Liquid Chromatography. Methods Mol. Biol. 2019, 2030, 131-142. 
49. Xiong, W.; Zhao, J.; Wang, L.; Jiang, X. UPLC-MS/MS method for the determination of tobacco-specific biomarker NNAL, tamoxifen and its main metabolites in rat plasma. Biomed. Chromatogr. 2017, 31, e3890. [CrossRef] [PubMed]

50. Goniewicz, M.L.; Eisner, M.D.; Lazcano-Ponce, E.; Zielinska-Danch, W.; Koszowski, B.; Sobczak, A.; Havel, C.; Jacob, P.; Benowitz, N.L. Comparison of Urine Cotinine and the Tobacco-Specific Nitrosamine Metabolite 4-(Methylnitrosamino)-1-(3Pyridyl)-1-Butanol (NNAL) and Their Ratio to Discriminate Active From Passive Smoking. Nicotine Tob. Res. 2011, 13, 202-208. [CrossRef]

51. Thomas, J.L.; Schreier, M.; Luo, X.; Lowry, S.; Hennrikus, D.; An, L.; Wetter, D.W.; Ahluwalia, J.S. Promoting Smoke-Free Homes Through Biomarker Feedback Documenting Child Exposure to Tobacco Toxins: Protocol for a Randomized Clinical Trial. JMIR Res. Protoc. 2019, 8, e12654. [CrossRef]

52. Kassem, N.O.; Daffa, R.M.; Liles, S.; Jackson, S.R.; Kassem, N.O.; Younis, M.A.; Mehta, S.; Chen, M.; Jacob, P., 3rd; Carmella, S.G.; et al. Children's exposure to secondhand and thirdhand smoke carcinogens and toxicants in homes of hookah smokers. Nicotine Tob. Res. 2014, 16, 961-975. [CrossRef]

53. Garcia-Perez, I.; Lindon, J.C.; Minet, E. Application of CE-MS to a metabonomics study of human urine from cigarette smokers and non-smokers. Bioanalysis 2014, 6, 2733-2749. [CrossRef] [PubMed]

54. Seow, W.J.; Shu, X.O.; Nicholson, J.K.; Holmes, E.; Walker, D.I.; Hu, W.; Cai, Q.; Gao, Y.; Xiang, Y. Association of Untargeted Urinary Metabolomics and Lung Cancer Risk among Never-Smoking Women in China. JAMA Netw. Open 2019, 2, e1911970. [CrossRef] [PubMed]

55. Wei, R.; Wang, J.; Su, M.; Jia, E.; Chen, S.; Chen, S.; Chen, T.; Ni, Y. Missing Value Imputation Approach for Mass Spectrometrybased Metabolomics Data. Sci. Rep. 2018, 8, 663. [CrossRef] [PubMed] 